Check for updates

Cite this: RSC Adv., 2019, 9, 7885

Received 21st December 2018

Accepted 4th March 2019

DOI: $10.1039 / \mathrm{c} 8 \mathrm{ra10467k}$

rsc.li/rsc-advances

\title{
Accurate, hysteresis-free temperature sensor for health monitoring using a magnetic sensor and pristine polymer $\uparrow$
}

\author{
Wooseong Jeong, (D) Mijin Kim, Jae-Hyun Ha, Nora Asyikin Binti Zulkifli, \\ Jung-Il Hong, D CheolGi Kim and Sungwon Lee*
}

\begin{abstract}
Herein, a novel temperature sensor is proposed employing a planar Hall resistance (PHR) magnetic sensor, a magnet sheet, and an expanding polymer. It features a tunable temperature range, high linear sensitivity, accuracy, and cycling stability through 1000 cycles, and no thermal hysteresis. This new sensor is promising as a body temperature sensor.
\end{abstract}

\section{Introduction}

Advances in science and technology have profoundly affected human lives, as exemplified by the increase in our average life expectancy. One way to extend life expectancy is to prevent accidents and emergency situations; another is to continuously monitor human health. To forestall unexpected health problems, accurately measuring body signals in real time is essential. Physical signals such as body temperature, blood pressure, heart rate, respiratory rate, and other biopotentials are important parameters for health monitoring. Accordingly, various physical sensors have recently been developed to satisfy the need for ubiquitous health monitoring systems..$^{1-3}$ In particular, physical sensors with bio-compatibility, ${ }^{4,5}$ conformal contact, ${ }^{6}$ stretchability, ${ }^{7}$ and other mechanical properties have been developed for long-term health monitoring: ${ }^{8}$

Body temperature is a particularly crucial marker of human health, as mammals tend to maintain homeostasis via the autonomic nervous system. However, body temperature can be affected by food poisoning, flu, inflammation, or heatstroke, especially in infants and the elderly. Hence, various sensors have been developed to monitor body temperature. ${ }^{9-13}$ Traditionally, pyroelectric detectors, thermistors, and resistive temperature detectors have been used. Recently, for wearable health-monitoring applications, diverse types of temperature sensors embedded in flexible and stretchable polymer substrates have been fabricated. In particular, electroconductive temperature sensors have been reported. These comprise a mixture of polymer and conductive particles and operate via the percolation threshold effect. These temperature

Department of Emerging Materials Science, Daegu Gyeongbuk Institute of Science \& Technology (DGIST), 333 Techno Jungang-daero, Hyeonpung-myeon, Dalseong-gun, Daegu 711-873, Korea.E-mail: swlee@dgist.ac.kr

$\dagger$ Electronic supplementary information (ESI) available. See DOI: 10.1039/c8ra10467k sensors have demonstrated outstanding properties, such as tunable temperature ranges and high sensitivities. For example, Bao's group developed a highly sensitive temperature sensor consisting of a Ni-microparticle-filled binary polymer composite. ${ }^{9}$ This sensor exhibited resistivity changes of six orders of magnitude upon a change in temperature of only $5{ }^{\circ} \mathrm{C}$. Someya's group developed similarly sensitive temperature sensors based on composites of semicrystalline acrylate polymers and graphite. ${ }^{10}$ These sensors could measure multipoint thermal data in real time, thus facilitating the successful measurement of dynamic temperature changes in the lung.

Nevertheless, electroconductive temperature sensors typically exhibit thermal hysteresis and inaccuracy $\left( \pm 3.1^{\circ} \mathrm{C}\right)$ during repeated heating and cooling, owing to the difficulty in controlling the dispersion state of the conductive particles in the polymer matrix. The conductive particles tend to aggregate during temperature cycling. In this study, we present a new concept for an extremely reproducible temperature sensor with high accuracy and no hysteresis to overcome these issues. To the best of our knowledge, this is the first attempt to fabricate a bio-sensor for health monitoring using a magnetic sensor and a polymer matrix.

\section{Experimental}

\section{Sensor fabrication}

A planar Hall resistance (PHR) magnetic sensor was selected owing to its high linearity, near-zero magnetic fields, low thermal drift, high signal-to-noise ratio, and high sensitivity. ${ }^{14,15}$ Semicrystalline polyethylene glycol (PEG, average molecular weight $M_{\mathrm{w}}=1000$ ) was selected as the polymer, as its volume considerably expands near body temperature (around 35-42 ${ }^{\circ} \mathrm{C}$ ), thus providing reversible volume control. However, before integrating the sensor into a wearable platform such as a skinattachable substrate, we first fabricated and analyzed its feasibility on a silicon wafer. 
To form the PHR sensor, layers of Ta/NiFe/IrMn/Ta (5/10/10/ $5 \mathrm{~nm}$ ) were deposited onto a silicon dioxide wafer by DC magnetron sputtering through a shadow mask. We optimized the thickness of the magnetic layers according to the magnetic properties and thickness dependence of the PHR sensor. ${ }^{15-17} \mathrm{~A}$ gold electrode was then sputtered onto the PHR sensor. PEG $\left(M_{\mathrm{w}}=600,1000\right.$, and 2000, Alfa Aesar) was molded onto the sensor using a polydimethylsiloxane (PDMS) mold to form the volume expansion material. A uniformly thick Parylene layer is crucial to secure the reversible temperature response, as discussed in the ESI. $\uparrow$ Thus, to withstand the volume expansion and maintain the shape of the polymer, a $3 \mu \mathrm{m}$-thick encapsulation layer of Parylene C (Daisan Kasei Co. Ltd, Japan) was deposited around the polymer and magnetic sensor using chemical vapor deposition (CVD). A commercial anisotropic magnet sheet (4.5 mm diam., $500 \mu \mathrm{m}$ height, JL Magnet, Korea) was affixed to the top of the molded PEG. Fig. S1 of the ESI $\dagger$ presents additional fabrication details.

\section{Characterization of polymer materials}

The specific volume expansion ratio was obtained using a gravity density cup. The material temperature was saturated in an oven for $4 \mathrm{~h}$ at each step. The melting temperature was measured by differential scanning calorimetry (DSC, Discovery DSC, TA Instruments) under a nitrogen atmosphere. The average heights of the molded PEG1000 and cross-sectional images were obtained with a 3D laser microscope (VK-X250, Keyence).

\section{Device characterization}

Transmission electron microscopy (TEM) images were obtained using a Hitachi HF-3300 TEM. The planar Hall effect (PHE) voltage profiles of the sensors were recorded using a multimeter (34401A, Hewlett Packard) with a magnetic field interval of \pm 220 Oe with custom LabVIEW programs. The current was applied using a multimeter (2400 SourceMeter, Keithley), and the magnetic field strength of the magnet sheet was recorded using a Gauss meter (Model 5080 Gauss/Tesla-meter, F. W. Bell, USA). The electrical properties of the temperature sensor were characterized using another multimeter with custom LabVIEW programs, applying a current of $50 \mathrm{~mA}$. The temperature was controlled using a Peltier device (TEC1-12706, JS Tech, Korea) through a programmable power supply (Model 2200-30-5, Keithley).

\section{Results and discussion}

As depicted schematically in Fig. 1, our temperature sensor consisted of a polymer sandwiched between a magnetic sensor and a commercial magnet sheet. The pristine polymer underwent precise changes in volume when exposed to external heat, while its shape was maintained by an encapsulation layer. As the polymer expanded and contracted, the distance between the magnet and the extremely sensitive magnetic sensor changed, causing minute variations in the magnetic field, which were detected by the sensor.

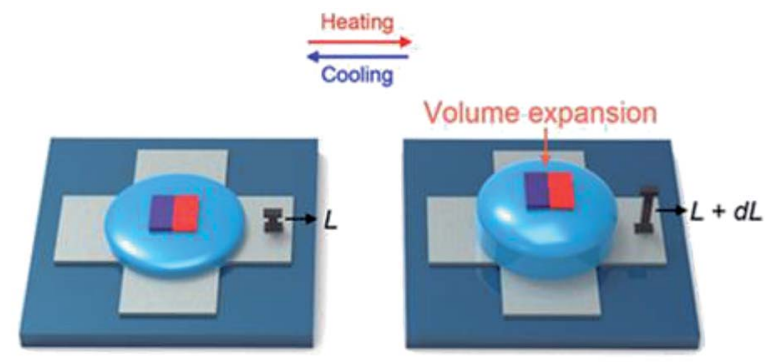

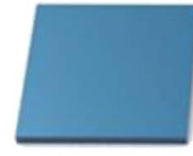

Substrate

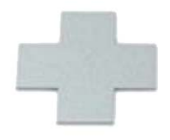

Magnetic sensor

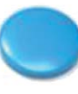

Polymer

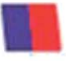

Magnet
Fig. 1 Schematic of the magnetic temperature sensor showing the volume expansion of the polymer upon heating.

Fig. 2a schematically depicts the PHR sensor structure. A field-emission TEM image of the Ta/NiFe/IrMn/Ta layers is shown in Fig. 2b. The Ta layers prevented oxidation and increased adhesion between the substrate and magnetic layer.

The magnetic layer, comprising nickel ferrite (ferromagnet) and iridium manganese (antiferromagnet) layers, generated the PHE voltage $\left(V_{\text {PHE }}\right)$. Fig. 2c shows the $V_{\text {PHE }}$ profiles obtained from 15 sensors when a current of $50 \mathrm{~mA}$ was applied. The sensors showed almost identical output voltages under a given magnetic field, indicating high reproducibility. The $V_{\mathrm{PHE}}$ profiles were recorded using a magnetoresistance measurement system and calculated theoretically as follows: ${ }^{17}$

$$
V_{\mathrm{PHE}}=\left(\left(I\left(\rho^{\|}-\rho^{\perp}\right)\right) / t\right) \sin \Theta \cos \Theta,
$$

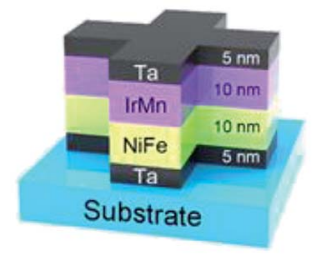

C

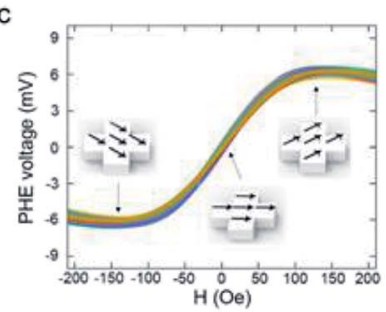

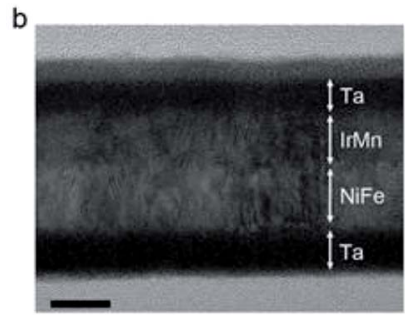

d

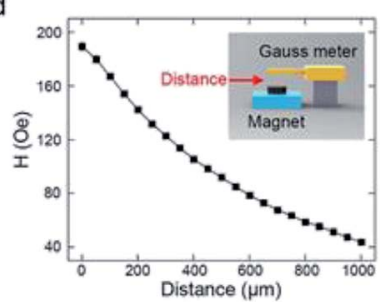

Fig. 2 Characteristics of the magnetic sensor and magnet sheet. (a) Schematic of the layered structure of the PHR sensor. (b) Crosssectional FE-TEM image of the PHR sensor (scale bar $=10 \mathrm{~nm}$ ). (c) PHE voltage profiles of $15 \mathrm{PHR}$ sensors, indicating high precision when a current of $50 \mathrm{~mA}$ was applied. (d) Magnetic field strength of the aligned magnet sheet as a function of the distance between the magnet and Gauss meter. 
where $I$ is the active current; $\rho^{\|}$and $\rho^{\perp}$ are the parallel and perpendicular resistivities, respectively; $t$ is the thickness of the active layer; and $\Theta$ is the angle between the magnetization direction and exchange bias field. The generation of the $V_{\mathrm{PHE}}$ can be explained in accordance with the anomalous Hall effect (Fig. S2 $\dagger$ ). ${ }^{18,19}$

To apply a magnetic field to the PHR sensor, a commercial anisotropic magnet sheet was used. According to the BiotSavart law, the strength of a magnetic field depends on angle and distance. ${ }^{20}$ Fig. $\mathrm{S} 3 \dagger$ shows the in-plane orientation and its dependence on distance. The magnet sheets were identically aligned onto the polymer layer throughout the experiments in this study. Their magnetic field strength was measured as a function of distance using a Gauss meter (Fig. 2d). A linear relationship was extracted from Fig. $2 \mathrm{c}$ and $\mathrm{d}$ and is presented in Fig. S4. $\dagger$ When the distance between the PHR sensor and magnet sheet was $400-900 \mu \mathrm{m}$, the PHE voltage exhibited excellent linearity, with a sensitivity of $4.58 \mu \mathrm{V} \mu \mathrm{m}^{-1}$. A linear relationship between the magnetic field and distance is important for optimum sensor design; hence, this linear range was used in the design to attain the best performance.

The thickness of the initial polymer layer was $\sim 550 \mu \mathrm{m}$. Semicrystalline PEG was used, a sizeable volume expansion is associated with its phase change from solid to liquid at the melting point $\left(T_{\mathrm{m}}\right)$. The maximum sensitivity range of the proposed temperature sensor could be easily tuned by changing the PEG molecular weight. Fig. 3a shows DSC thermograms of PEG600 $\left(M_{\mathrm{w}}=600, T_{\mathrm{m}}=17-23^{\circ} \mathrm{C}\right)$, PEG1000 $\left(M_{\mathrm{w}}=1000, T_{\mathrm{m}}=\right.$ $\left.35-40{ }^{\circ} \mathrm{C}\right)$, and PEG2000 $\left(M_{\mathrm{w}}=2000, T_{\mathrm{m}}=53-55{ }^{\circ} \mathrm{C}\right)$. Fig. S5 $\dagger$ shows the molecular weight distributions of PEG600, PEG1000, and PEG2000. For use in a body-temperature $\left(35-42{ }^{\circ} \mathrm{C}\right)$ sensor, the volume expansion, $V_{\text {exp }}$, was calculated as

$$
V_{\exp }(\%)=\left(V-V_{0}\right) / V_{0} \times 100,
$$
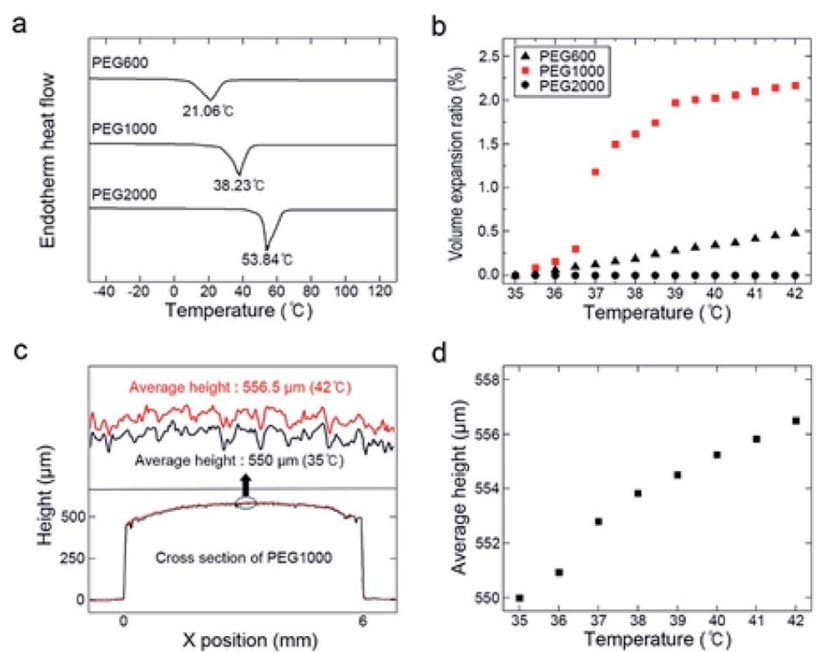

Fig. 3 Characteristics of the polymer materials. (a) DSC curves indicating the melting temperature ranges and (b) normalized specific volume expansion ratios of PEG600, PEG1000, and PEG2000. (c) Cross-sectional height of molded PEG1000 at 35 and $42{ }^{\circ} \mathrm{C}$. (d) Average height of molded PEG1000 as a function of temperature. where $V$ and $V_{0}$ are the volumes at the set and initial temperatures, respectively. Owing to its suitable $T_{\mathrm{m}}$, PEG1000 showed significantly higher volume expansion $\left(V_{\exp } \approx 2.2 \%\right)$ across the selected sensitivity range $\left(35-42{ }^{\circ} \mathrm{C}\right)$ than PEG600 or PEG2000 (Fig. 3b) and was therefore applied in the body-temperature sensor.

PEG1000 was molded into a cylinder using a PDMS mold. To maintain its form throughout the phase change, a Parylene C encapsulation layer was deposited onto the PEG1000 and PHR sensor (Fig. S6a and $\mathrm{b} \dagger$ ). During repeated heating and cooling, the cylindrical shape was somewhat transformed into a droplet shape due to surface energy minimization and the phase transition. However, after a few heating and cooling cycles, the shape remained uniform upon temperature changes. After this shape stabilized, we were able to quantifiably measure the displacement of the molded PEG1000 in response to temperature using 3D confocal laser microscopy. Fig. 3c shows the cross-sectional height profiles of the molded PEG1000 at 35 and $42{ }^{\circ} \mathrm{C}$. The 3D structure is also illustrated in Fig. S6c and d. $\dagger$ Fig. 3d shows the average height of the molded PEG1000 between 35 and $42{ }^{\circ} \mathrm{C}$ at intervals of $1{ }^{\circ} \mathrm{C}$. A linear height increase with no fluctuations was clearly observed.

As a final step in sensor fabrication, the magnet sheet was attached to the molded PEG1000. A digital photograph of the temperature sensor is shown in Fig. 4a. To analyze the dependence of $V_{\mathrm{PHE}}$ on temperature, the output signals were normalized. The variation in the output signal was calculated as
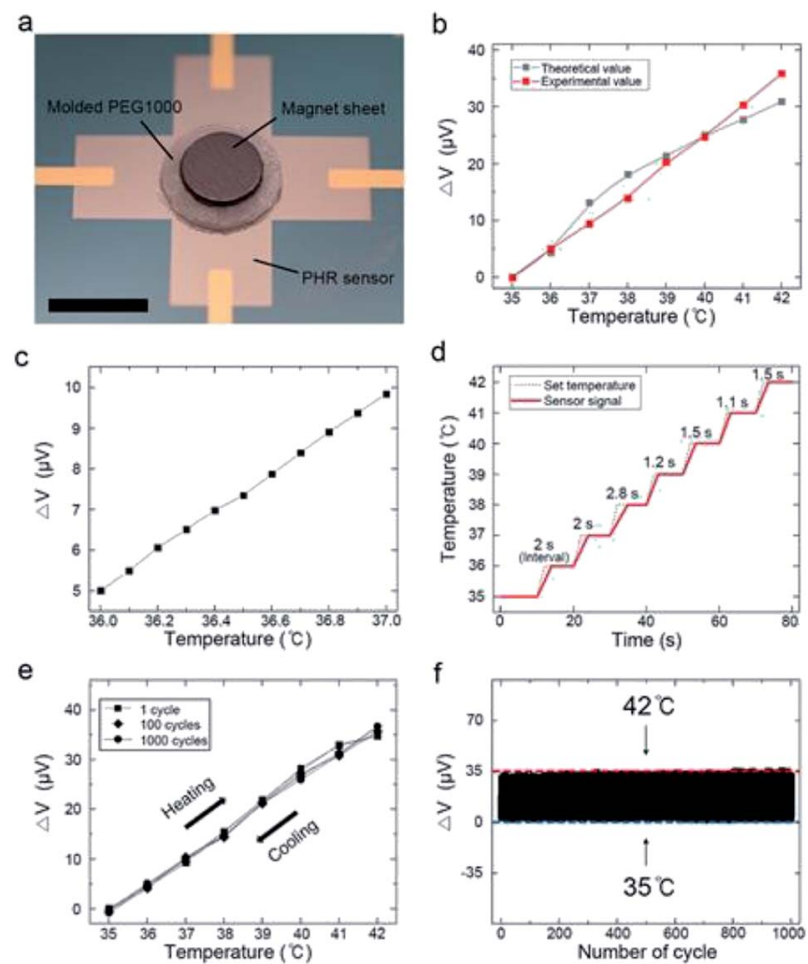

Fig. 4 Normalized electrical performance of the novel temperature sensor. (a) Photograph of the temperature sensor on $\mathrm{SiO}_{2}$ wafer (scale bar $=5 \mathrm{~mm}$ ). (b) Theoretical and experimental values of output voltage. (c) Output voltage from 36 to $37^{\circ} \mathrm{C}$ at intervals of $0.1^{\circ} \mathrm{C}$. (d) Response time of the sensor. (e) Hysteresis loop of the sensor, indicating no temperature deviation. (f) Cycling test in the temperature range of 35 to $42{ }^{\circ} \mathrm{C}$. 


$$
\Delta V_{\mathrm{PHE}}=V_{\mathrm{PHE}, \text { initial }}-V_{\mathrm{PHE}, \text { final }}
$$

where $\Delta V_{\mathrm{PHE}}$ was the variation in the PHE voltage, and $V_{\mathrm{PHE}}$ initial and $V_{\mathrm{PHE}}$, final were the PHE voltages at $35{ }^{\circ} \mathrm{C}$ and the set temperature, respectively. Additionally, because the PHR sensor contained metals, the temperature-dependent variation in the output signal was determined (Fig. S7 $\dagger$ ). The normalized output signals excluded this factor to show only the magnetic-fieldstrength-dependent variation in $V_{\mathrm{PHE}}$ in response to temperature.

To verify the experimentally obtained values, we calculated the theoretical output voltage values using the generation of $V_{\text {PHE }}$ in response to the magnetic field (Fig. 2c), the variation in the magnetic field depending on distance (Fig. 2d), and the displacement of the polymer at $35-42{ }^{\circ} \mathrm{C}$ (Fig. 3d). Fig. $4 \mathrm{~b}$ compares the theoretical and experimental output signals of the temperature sensor at $35-42{ }^{\circ} \mathrm{C}$. These signals differed slightly, because the PEG1000 was molded, and the magnet sheet was aligned manually. Nevertheless, the experimental values showed excellent linearity not only in the range of $35-42{ }^{\circ} \mathrm{C}$ but also over smaller temperature ranges with intervals of $0.1^{\circ} \mathrm{C}$, as shown in Fig. 4c. Remarkably, this indicated that our sensor precisely detected a displacement of $92.8 \mathrm{~nm}$ between the magnet and magnetic sensor.

Fig. $4 \mathrm{~d}$ shows that the average response time of the sensor was $1.73 \mathrm{~S}^{\circ} \mathrm{C}^{-1}$. The response time was slightly longer at $\sim 38^{\circ} \mathrm{C}$ because of the phase transition. The cycling stability was evaluated from 35 to $42{ }^{\circ} \mathrm{C}$ over 1000 cycles. Fig. 4e shows the thermal hysteresis loops of the 1st, 100th, and 1000th cycles under continuous heating and cooling. Notably, almost no hysteresis was observed, although the sensitivity was identical throughout the measurements. Furthermore, the output signals of the temperature sensor did not show any distinct degradation (Fig. 4e and f). Additional information about the cycling tests is shown in Fig. S8. $\dagger$

To function well in the temperature sensor, the volume expansion of the polymeric system must be reversible. The Parylene encapsulation layer is important for accommodating the reversible volume expansion, as illustrated in Fig. S9. $\dagger$ Interestingly, the volume increase was observed only along the $y$-axis $(1.18 \%)$, whereas the dimensions along the $x$ - and $z$-axes remained constant. Thus, the encapsulation layer did not delaminate upon expansion and contraction of the polymer.

Owing to the properties of the PHR sensor and the polymeric system, this temperature sensor exhibited unprecedented repeatability, high sensitivity, accuracy, and no thermal hysteresis, thereby demonstrating its potential as a temperature sensor embedded in a polymer matrix. Additionally, the PHE voltage could be measured accurately because of the high sensitivity of the PHR sensor, which enabled the detection of sub-micrometer-scale displacement of the magnet.

\section{Conclusions}

In summary, we report the design and development of a novel temperature sensor comprising a magnetic sensor, magnet sheet, and expanding polymer. We established a new design employing reversible volume expansion to control the magnetic field strength during temperature changes. The present study demonstrates the possibility of detecting body temperature, an important vital sign, by applying magnetic sensors and polymers. Although this new biosensor-fabrication concept for health-monitoring systems is demonstrated here for temperature sensing, other types of physical sensors can be envisioned that would exploit other functional polymers that are not thermally sensitive. For example, stimuli-sensitive polymers such as elastomers could be utilized as pressure or strain sensors by detecting displacement between a magnetic sensor and magnet when such stimuli are applied. Additionally, humidity-sensitive polymers such as hydrogels could be utilized in humidity sensors that detect displacements as a function of humidity. Moreover, this approach could be extended to the fabrication of wearable magnetic sensors for health-monitoring applications., ${ }^{4,5,21}$ To measure signals from the body in real time, our future work will focus on improving the flexibility and stretchability of the temperature sensor.

\section{Author contributions}

W. J. fabricated the temperature sensor and analyzed the data. M. K., J. Ha, and N. A. B. Z fabricated the PHR sensor and measured the PHE voltages. J. Hong and C. K. provided advice about magnetic sensors and magnetism. W. J. and S. L. planned the experiments and prepared the manuscript.

\section{Conflicts of interest}

There are no conflicts to declare.

\section{Acknowledgements}

This research was supported by the National Research Foundation of Korea (NRF-2017R1D1A1B03035194 \& 2018R1A5A1025511) and the DGIST R\&D Program of the Ministry of Science, ICT and Future Planning (18-BT-02).

\section{References}

1 D.-H. Kim, N. Lu, R. Ghaffari, Y.-S. Kim, S. P. Lee, L. Xu, J. Wu, R.-H. Kim, J. Song, Z. Liu, J. Viventi, B. de Graff, B. Elolampi, M. Mansour, M. J. Slepian, S. Hwang, J. D. Moss, S.-M. Won, Y. Huang, B. Litt and J. A. Rogers, Nat. Mater., 2011, 10, 316.

2 D. Son, J. Lee, S. Qiao, R. Ghaffari, J. Kim, J. E. Lee, C. Song, S. J. Kim, D. J. Lee, S. W. Jun, S. Yang, M. Park, J. Shin, K. Do, M. Lee, K. Kang, C. S. Hwang, N. Lu, T. Hyeon and D.-H. Kim, Nat. Nanotechnol., 2014, 9, 397.

3 T. Q. Trung and N.-E. Lee, Adv. Mater., 2016, 28, 4338.

4 A. Miyamoto, S. Lee, N. F. Cooray, S. Lee, M. Mori, N. Matsuhisa, H. Jin, L. Yoda, T. Yokota, A. Itoh, M. Sekino, H. Kawasaki, T. Ebihara, M. Amagai and T. Someya, Nat. Nanotechnol., 2017, 12, 907. 
5 S. Lee, A. Reuveny, J. Reeder, S. Lee, H. Jin, Q. Liu, T. Yokota, T. Sekitani, T. Isoyama, Y. Abe, Z. Suo and T. Someya, Nat. Nanotechnol., 2016, 11, 472.

6 R. C. Webb, A. P. Bonifas, A. Behnaz, Y. Zhang, K. J. Yu, H. Cheng, M. Shi, Z. Bian, Z. Liu, Y.-S. Kim, W.-H. Yeo, J. S. Park, J. Song, Y. Li, Y. Huang, A. M. Gorbach and J. A. Rogers, Nat. Mater., 2013, 12, 938.

7 T. Q. Trung and N.-E. Lee, Adv. Mater., 2017, 29, 1603167.

8 J. A. Rogers, T. Someya and Y. Huang, Science, 2010, 327, 1603.

9 J. Jeon, H.-B.-R. Lee and Z. Bao, Adv. Mater., 2013, 25, 850.

10 T. Yokota, Y. Inoue, Y. Terakawa, J. Reeder, M. Kaltenbrunner, T. Ware, K. Yang, K. Mabuchi, T. Murakawa, M. Sekino, W. Voit, T. Sekitani and T. Someya, Proc. Natl. Acad. Sci. U. S. A., 2015, 112, 14533.

11 T. Q. Trung, S. Ramasundaram, B.-U. Hwang and N.-E. Lee, Adv. Mater., 2016, 28, 502.

12 S. Harada, K. Kanao, Y. Yamamoto, T. Arie, S. Akita and K. Takei, ACS Nano, 2014, 8, 12851.
13 L. Gao, Y. Zhang, V. Malyarchuk, L. Jia, K.-I. Jang, R. C. Webb, H. Fu, Y. Shi, G. Zhou, L. Shi, D. Shah, X. Huang, B. Xu, C. Yu, Y. Huang and J. A. Rogers, Nat. Commun., 2014, 5, 4938.

14 B. Sinha, T. Q. Hung, T. S. Ramulu, S. Oh, K. Kim, D.-Y. Kim, F. Terki and C. G. Kim, J. Appl. Phys., 2013, 113, 063903.

15 N. T. Thanh, L. T. Tu, N. D. Ha, C. O. Kim and C. G. Kim, J. Appl. Phys., 2007, 101, 053702.

16 S. Anandakumar, V. S. Rani, S. Oh and C. G. Kim, Thin Solid Films, 2010, 519, 1020.

17 T. Q. Hung, S. Oh, B. Sinha, J.-R. Jeong, D.-Y. Kim and C. G. Kim, J. Appl. Phys., 2010, 107, 09E715.

18 J. M. Luttinger, Phys. Rev., 1958, 112, 739.

19 A. Crepieux and P. Bruno, Phys. Rev. B: Condens. Matter Mater. Phys., 2001, 64, 014416.

20 G. Cavalleri, G. Spavieri and G. Spinelli, Eur. J. Phys., 1996, 17, 205.

21 S. Lee, Y. Inoue, D. M. Kim, A. Reuveny, K. Kuribara, T. Yokota, M. Sekino, T. Sekitani, Y. Abe and T. Someya, Nat. Commun., 2014, 5, 5898. 